\title{
Evaluación y construcción de un perfil de competencias profesionales en medicina interna
}

\author{
Evaluation and construction of a profile of \\ professional competences in internal medicine
}

\author{
Análida Elizabeth Pinilla, Fidel Antonio Cárdenas \\ - Bogotá D.C. (Colombia)
}

\section{Resumen}

Objetivo: caracterizar el perfil de desempeño del médico internista según las competencias profesionales propuestas y establecer las relaciones con el perfil de formación actual en esta especialidad en Colombia.

Diseño del estudio: estudio mixto, transversal, durante 11 meses; con consentimiento informado y Comité de Ética.

Participantes: directivos, internistas, internistas-docentes, residentes. Intervenciones: entrevistas en profundidad, encuesta y análisis de programas. Encuesta anónima, según el análisis cualitativo de entrevistas. Triangulaciones para validez y confiabilidad por fuentes (literatura, participantes, programas), instrumentos (entrevista, encuesta, rejilla para análisis de programas) y tipo de participantes.

Análisis: cualitativo Atlas.ti 5.0, cuantitativo Excel 2007.

Resultados: entrevistados 45, encuestados 190 y programas analizados 2, para proponer un perfil de formación por competencias profesionales con enfoque pedagógico socio-constructivista.

Este perfil propuesto reconfirma las competencias profesionales de la literatura pero surgieron unas nuevas: 1. Específicas nucleares emergentes, que dan la identidad deontológica o razón de ser del internista, son las capacidades para integrar problemas del paciente, organizar conceptos de subespecialistas, ser experto en enfermedades prevalentes, ser soporte de especialistas médico-quirúrgicos, actuar en diferentes niveles de atención y escenarios. 2. Clínicas clásicas y otras nuevas como enfatizar la promoción de la salud y prevención de la enfermedad. 3. Transversales emergentes: comunicativas, profesionalismo, administrativas, pedagógicas, en investigación.

Conclusión: el perfil de formación del residente continúa centrado en objetivos y rotaciones fragmentadas en medicina interna y subespecialidades; se infiere un enfoque empírico en competencias profesionales clínicas y el conocimiento médico, mediante los convenios docencia-servicio; hay carencia del desarrollo formal de competencias transversales y específicas nucleares. (Acta Med Colomb 2014; 39: 165-173).

Palabras clave: atención al paciente, medicina interna, educación basada en competencias, educación médica.

\section{Abstract}

Objective: characterize the performance profile of the internal medicine doctor according to the proposed professional competences and establish relationships with the profile of current training in this specialty in Colombia.

Study design: joint cross-sectional study, for 11 months, with informed consent and Ethics Committee.

Participants: managers, internists, teaching internists, residents.

Interventions: thorough interviews, survey and programs analysis. Anonymous survey, according to the qualitative analysis of interviews. Triangulations for validity and reliability by sources (literature, participants, programs), instruments (interview, survey, grid analysis programs) and type of participants.

Analysis: Atlas.ti 5.0 qualitative, quantitative Excel 2007.

Dra. Análida Elizabeth Pinilla Roa: Internista. Formación en nutrición y diabetes. MSc. en Educación con Énfasis en Docencia Universitaria. Especialista en Evaluación y Construcción de Indicadores de Gestión para la Educación Superior. PhD en Educación. FACP. Coordinadora Grupo de Apoyo Pedagógico y Formación Docente. Profesora Asociada Departamento de Medicina Interna, Facultad de Medicina. Universidad Nacional de Colombia; Dr. Fidel Antonio Cárdenas Salgado: Licenciado en Educación con estudios principales en Química. MSc en Enseñanza de Ciencias y Matemáticas. PhD en Química. Profesor Titular y Coordinador Sistema de Formación Avanzada Universidad Pedagógica Nacional. Bogotá D.C. (Colombia). Correspondencia. Dra. Análida Elizabeth Pinilla Roa. Bogotá D.C. (Colombia) Correo electrónico:aepinillar@unal.edu.co Recibido: 06/VI/2013 Aceptado: 03/III/2014 
Results: 45 interviewed, 190 polled and two programs analyzed to propose a profile of training by professional competences with socio-constructivist pedagogical approach.

This proposed profile reconfirms the professional competences of literature but new ones arose:

1. Specific emerging nuclear, which give the deontological identity or reason for being an internist, are the capacities to integrate patient problems, organize concepts of subspecialities, be skilled in prevalent diseases, be support for medical and surgical specialists and act in different levels of care and scenarios.

2. Classic clinics and new ones like emphasizing health promotion and disease prevention .

3. Cross-emerging: communicative, professionalism, administrative, pedagogic, in research.

Conclusion: the profile of resident training continues to focus on objectives and fragmented rotations in internal medicine and subspecialities; an empirical approach in clinical skills and medical knowledge through teaching-service agreements is inferred; there is lack of formal development of cross and nuclear specific competences. (Acta Med Colomb 2014; 39: 165-173).

Keywords: patient care, internal medicine, competency-based education, medical education.

\section{Introducción}

En concordancia con los requerimientos nacional e internacional, es crucial que en los programas de la especialidad de medicina interna del país, los médicos desarrollen diversas competencias profesionales $(\mathrm{CP})$ que van más allá del aprendizaje y la apropiación del conocimiento médico de la especialidad para atender un paciente y de unas habilidades para ejecutar procedimientos específicos (1). En este sentido, un residente en medicina interna más que replicar saberes y conceptos preestablecidos por otros o plasmados en la literatura, tiene que ser un profesional que analiza, crítica y reconstruye el conocimiento con el fin de favorecer la investigación y el cambio cultural (2). En Colombia la especialidad en medicina interna surgió a comienzos de los años sesenta y, transcurrido más de medio siglo, del funcionamiento de diferentes programas de esta especialidad, continúa pendiente un referente teórico y pedagógico sistematizado para la formación de un médico internista. Este proyecto aporta a la identidad deontológica o razón de ser de este especialista y su relevancia para cualquier sistema de salud dado que todo internista debe responder cabalmente al paciente, su familia y la sociedad.

En esta investigación sobre la formación de médicos residentes, en la especialidad de medicina interna en Colombia, se plantean tres perfiles de formación por medio del desarrollo de CP: el teórico de la literatura (Tabla 1); el perfil propuesto basado en el anterior y los aportes de entrevistados y encuestados; y el perfil actual de formación basado en el análisis de dos programas de formación. Finalmente se contrasta el perfil propuesto con el de formación actual, para explicitar las relaciones entre éstos.

Para comenzar es necesario precisar el concepto competencia en educación, en particular en el contexto de la educación médica en posgrado, que para el caso de la formación en especialidades médicas y quirúrgicas, se puntualiza como educación basada en CP con un enfoque pedagógico socio-constructivista interpretativo, el cual explica el enlace con las competencias académicas y las competencias laborales.
En este enfoque o modelo pedagógico, el concepto de competencia en educación médica, implica todo un conjunto de capacidades que desarrolla una persona, responsable y autónoma, mediante procesos conscientes para llegar a ser competente o idónea en su desempeño profesional. Las CP abarcan un conjunto de atributos interrelacionados e integrados: actitudes y valores o virtudes (meta-cognitivas y socioafectivas, saber-ser), conocimientos (dimensión cognitiva, saber) y habilidades y destrezas (dimensión motora, procedimientos, saber hacer, hacer sabiendo, entendiendo lo que se hace); así todo estudiante autónomo va desarrollándolas por procesos desde el pregrado, el posgrado y continúa haciéndolo durante la práctica profesional; se precisa al lector que este tema de la genealogía y desarrollo del concepto competencia y CP se escapa al objeto de este artículo (3-9).

El objetivo general de esta investigación fue caracterizar el perfil para el desempeño de un médico internista a partir de un conjunto de CP propuesto y establecer las relaciones con las competencias de formación actual de los estudiantes de esta especialidad. Se plantearon como objetivos específicos: identificar las $\mathrm{CP}$ de un médico internista según la literatura, proponer un perfil de $\mathrm{CP}$ para un médico internista en Colombia, explicitar el perfil de CP con el cual se están formando los médicos internistas en dos programas de medicina interna en Colombia y analizar las posibles relaciones entre el perfil propuesto y el perfil de formación actual de esta especialidad.

Tabla 1. Perfil teórico de competencias profesionales de un médico internista.

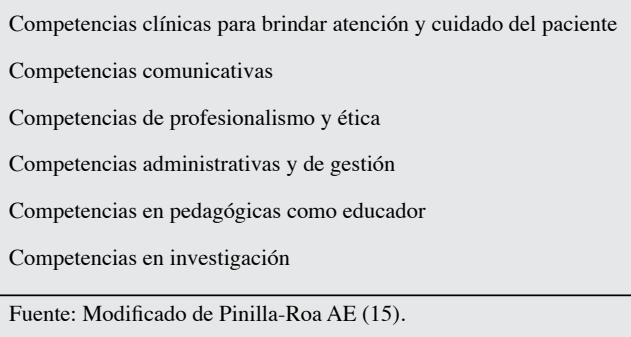




\section{Material y métodos}

Investigación de corte transversal, tradicionalmente llamada mixta y ahora denominada alternativa, con trabajo de campo durante 11 meses, el investigador alternativo comprende que la realidad social por estudiar es compleja y abarca elementos cuantitativos y cualitativos; así, se busca acabar con la dicotomía entre métodos y técnicas de recolección de la información (10). Se emplearon elementos de la investigación tradicional o cuantitativa, como: revisión de la literatura, encuesta y análisis documental de dos programas de medicina interna; de igual forma se emplearon elementos de la investigación cualitativa: entrevistas en profundidad con preguntas abiertas elaboradas a partir del perfil teórico de la literatura (Tabla 1), que se analizaron e interpretaron con apoyo del método de la teoría fundamentada $(11,12)$.

Los conceptos de confiabilidad y validez en esta postura epistemológica de la investigación alternativa, se fundamentan en la triangulación entendida como la contrastación de la información mediante diferentes fuentes, instrumentos, participantes, entre otros (13). La primera triangulación se hizo por fuentes: literatura, aporte de participantes y programas de medicina interna. La segunda triangulación, se consolidó por medio de los instrumentos: entrevistas en profundidad, encuesta (en línea y personal) y la rejilla para análisis de documentos de programas de medicina interna. Y, la tercera triangulación por el tipo de participantes: médicos directivos, médicos internistas docentes, coordinadores de programa, médicos internistas no docentes y médicos residentes. Por tanto el número de participantes en las entrevistas en profundidad se definió durante el proceso, cuando al analizarlas se evidenció que la información obtenida comenzó a ser repetitiva y redundante, es decir cuando apareció el punto de saturación $(11,13,14)$.

\section{Participantes}

- Directivos: profesionales que dirigen o contratan a internistas, como: directores científicos de entidades promotoras de salud (EPS), directivos de universidades (rector, decano, director de posgrado, coordinador de programa).

- Médico internista docente y coordinador: médico internista con función doble, ser docente de un programa de medicina interna y, a su vez, ser el coordinador del mismo.

- Médicos internistas docentes: internistas con vinculación a la academia como docentes en alguna universidad que ofrece el programa de la especialidad en medicina interna y con experiencia mayor o igual a tres años.

- Médicos internistas: en ejercicio o pensionados, sin vinculación a la docencia, es decir que se desempeñan en diferentes centros, clínicas, hospitales o EPS.

- Médico-residente: estudiantes de posgrado de diferentes programas de medicina interna.
Entrevistas. Se hizo el análisis e interpretación de las entrevistas transcritas, de manera literal y completa de todo lo enunciado; a cada entrevistado se le asignó un código, con el fin de proteger la identidad y mantener el anonimato, éste fue empleado para categorizar cada una de las respuestas.

Las entrevistas se analizaron mediante el programa Atlas.ti. Se creó una unidad hermenéutica con todos los documentos primarios que se construyeron de cada pregunta y las respuestas de cada uno de los 45 entrevistados (Tabla 2). Para el análisis cualitativo se realizó el proceso de codificación para resumir las respuestas y expandir las categorías del perfil de CP teórico, así, aparecieron categorías emergentes o inductivas (Tabla 1) (15); luego se organizaron los códigos en familias mediante una actividad reflexiva y dinámica al establecer relaciones entre códigos para generar las familias de CP de un médico internista $(16,17)$.

Encuesta. Para evaluar la consistencia del perfil de CP propuesto por los entrevistados y buscar la opinión de otros médicos internistas del país.

La construcción, validación del instrumento implicó un proceso hasta terminar en el instrumento por aplicar. La primera versión de la encuesta fue construida a partir del análisis e interpretación de las entrevistas; luego, fue revisada por pares académicos de ciencias de la salud (con y sin formación en educación y por dos pedagogos doctores en educación). Después se realizó la prueba final con 20 internistas, mediante entrevista personal individualizada, quienes adicionaron inquietudes de redacción y contenido que fueron plasmadas en el cuestionario definitivo (18).

Análisis de programas. Se buscó en Internet y en la $\mathrm{ACMI}^{\circledR}$ los programas que actualmente están funcionando en Colombia, se procedió a realizar un análisis general de todos los documentos encontrados en la web y se seleccionaron dos programas según: lo aprobado en el proyecto, la amplitud de la información en la web y la aceptación, a participar con la entrevista de los coordinadores. Para el análisis detallado del plan de estudios de estos programas se elaboró una rejilla.

Tabla 2. Documentos primarios a partir de cada pregunta de las entrevistas

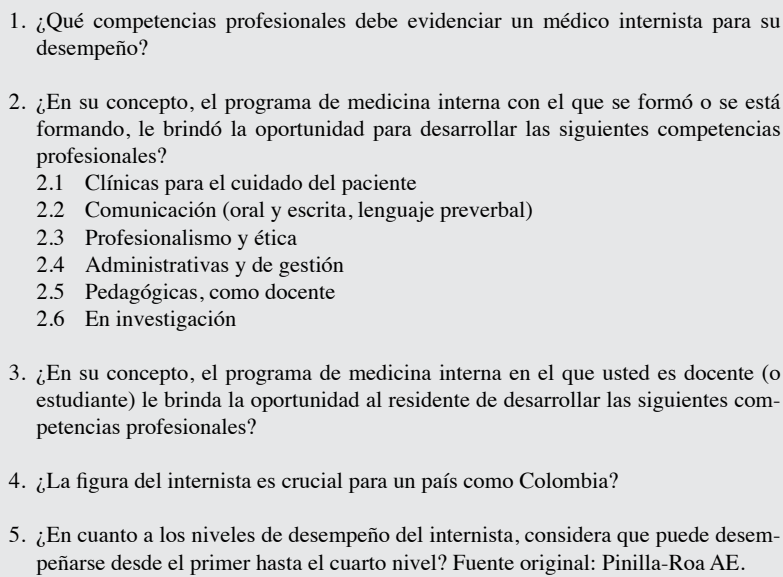

1. ¿Qué competencias profesionales debe evidenciar un médico internista para su desempeño?

2. ¿En su concepto, el programa de medicina interna con el que se formó o se está formando, le brindó la oportunidad para desarrollar las siguientes competencias profesionales?

2.1 Clínicas para el cuidado del paciente

2.2 Comunicación (oral y escrita, lenguaje preverbal)

2.3 Profesionalismo y ética

2.4 Administrativas y de gestión

2.5 Pedagógicas, como docente

2.6 En investigación

3. ¿En su concepto, el programa de medicina interna en el que usted es docente ( estudiante) le brinda la oportunidad al residente de desarrollar las siguientes competencias profesionales?

4. ¿La figura del internista es crucial para un país como Colombia?

5. ¿En cuanto a los niveles de desempeño del internista, considera que puede desempeñarse desde el primer hasta el cuarto nivel? Fuente original: Pinilla-Roa AE. 
Además, el análisis cuantitativo de la información demográfica de los entrevistados se realizó en el programa Excel 2007, al igual que el análisis e interpretación de la encuesta (18).

\section{Consideraciones éticas}

Este proyecto se ajustó a la normatividad para la investigación en salud según la Resolución 008430 de 1993 del Ministerio de Salud y las consideraciones éticas del consentimiento informado (19); una vez, la investigadora recibió la aprobación por el Consejo Académico del Doctorado en Educación (CADE) de la Universidad Pedagógica Nacional, procedió a solicitar la revisión y aprobación de los estamentos de la Facultad de Medicina de la Universidad Nacional de Colombia: Comité de investigación del Departamento de Medicina Interna, Comité de Ética y aval del Consejo de Facultad.

\section{Resultados}

Se realizó entrevista en profundidad a 45 profesionales: 11 directivos médicos, 11 médicos internistas, 13 médicos internistas docentes, ocho médicos-residentes y dos médicos internistas docentes coordinadores de programas de la especialidad en medicina interna; los participantes fueron de diferentes edades: 13 menores de 35 años, 14 entre 36-50 años, nueve entre 51-65 años y nueve mayores de 65 años (Figura 1); al igual que de diferentes años de graduación: 14 hasta 1976, 17 entre 1977-1993, siete entre 1994-2010 y los siete restantes eran residentes.

Para la encuesta en línea se invitaron a 998 internistas y residentes colombianos, en dos invitaciones personalizadas. De los que abrieron el correo la ubicación fue diversa: 421 en Colombia, 22 en Estados Unidos, seis en España, seis en Argentina, cinco en Francia, cuatro en Alemania y los restantes en otros países; respondieron la encuesta anónima, en línea 149 (14.93\% de invitados) y de forma personal 41 para un total de 190 profesionales.

Todo lo anterior condujo a la construcción social de un perfil de CP para un médico internista en Colombia, a partir de la triangulación entre el perfil teórico de la literatura, que fue enriquecido con aportes de los actores de la medicina interna (entrevistados y encuestados) para llegar al perfil propuesto (15). Luego esta triangulación se completó con el análisis de contenido de los documentos, de dos programas de la especialidad de medicina interna, para explicitar el perfil de formación actual. Finalmente se establecieron las relaciones entre el perfil propuesto de CP y el perfil de formación actual.

Se fueron reconfigurando las familias de $\mathrm{CP}$ con el fin de comprender la realidad actual de un internista competente o idóneo; por lo anterior, se continuó la identificación y la reconceptualizaron de códigos y a su vez, surgieron unos nuevos (inductivos o emergentes) que no se incluían inicialmente. En el perfil propuesto se confirmaron todas las $\mathrm{CP}$ del perfil teórico; sin embargo, éste fue enriquecido con nuevas
CP emergentes codificadas que sirvieron para conceptualizar y elaborar un perfil particular de $\mathrm{CP}$ de un médico internista para Colombia, el interés era captar la realidad de la práctica profesional actual de los médicos internistas.

No obstante, de acuerdo con el concepto de CP propuesto en esta investigación se reestructuraron las categorías que aparecen en la literatura, en particular la categoría de "competencias de conocimiento médico"; lo anterior porque el conocimiento es parte de todas las $\mathrm{CP}$ de un médico internista. El conocimiento médico que tiene un internista, se organizó en:

1. Procesos de aprendizaje: actualizar los conocimientos de forma permanente; saber aprender, desaprender y volver a aprender; contextualizar el conocimiento científico a cada paciente; identificar los límites entre el conocimiento en medicina interna y el de las subespecialidades para definir su acción.

2. Áreas de conocimiento específico: conceptos desde las ciencias básicas que se integran a las ciencias clínicas para brindar atención y cuidado a un paciente.

El perfil de formación para un residente propuesto por los participantes, está constituido por tres familias de CP que se diagraman en la Figura 2, a partir de las redes semánticas construidas en el programa Atlas.ti.

En primer lugar, las CP específicas clásicas o clínicas que sirven para brindar atención y cuidado al paciente: elaborar una historia clínica; realizar un examen físico completo y enfocado; construir un enfoque diagnóstico y terapéutico acorde a cada caso clínico; tener juicio clínico; saber hacer diferentes procedimientos de diagnóstico y tratamiento usuales (toracentesis, paracentesis, tomar un electrocardiograma, colocar un catéter central en arteria subclavia, entre otros) y emergente realizar un Doppler arterial de miembros inferiores para calcular el índice tobillo/brazo; además surgió como nueva $\mathrm{CP}$ enfatizar en la práctica de promoción de la salud y prevención de la enfermedad.

En segundo lugar se destaca una nueva familia de $\mathrm{CP}$ específicas nucleares, denominadas así en esta investigación, porque garantizan la identidad deontológica del médico internista: integrar todos los problemas de un paciente para

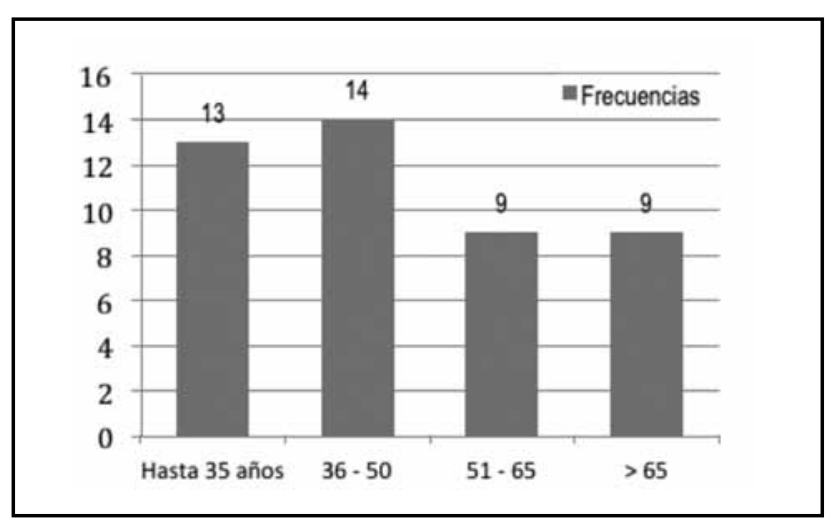

Figura 1. Distribución porcentual de los entrevistados según rangos de edad. 


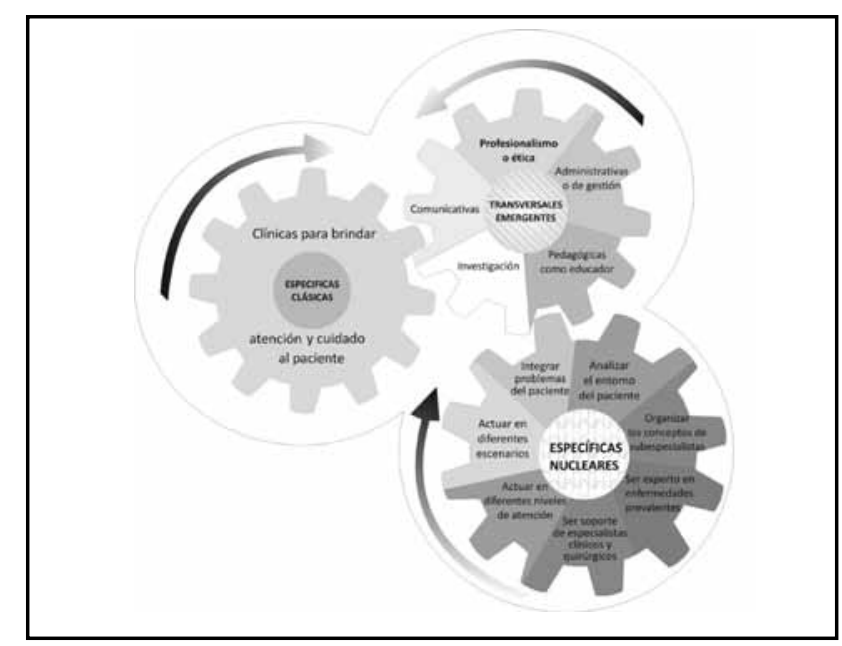

Figura 2. Perfil de competencias de un médico internista. Fuente original: Pinilla-Roa AE. Elaboración a partir de la teorización.

brindarle soluciones; analizar el entorno del paciente en lo social, económico, cultural para brindarle soluciones; organizar los conceptos de los médicos especialistas e internistas subespecialistas sobre un paciente para definir un diagnóstico y un tratamiento individualizado; ser experto en las enfermedades más prevalentes con el fin de resolver el mayor porcentaje de problemas de un paciente; ser soporte de los especialistas clínicos y quirúrgicos; asimismo actuar en diferentes niveles de atención y en diferentes escenarios (urgencias, consulta externa, hospitalización en pisos, cuidado intermedio, cuidado intensivo).

En tercer lugar las CP transversales, que son comunes a los diferentes profesionales de ciencias de la salud, pero que no están visibles en los programas, razón por la cual también se les denominó emergentes, éstas son:

Comunicativas: saber escuchar al paciente como persona; saber comunicar y explicar al paciente y sus familiares el diagnóstico y el tratamiento; liderar al equipo de trabajo para atender al paciente; saber comunicarse en público y con otros profesionales; saber comunicarse de forma escrita en las historias clínicas y publicaciones; y saber usar la tecnología de la información y la comunicación.

Profesionalismo o ética: tener sensibilidad por el paciente como persona; enfatizar en la persona más allá de la enfermedad; comprender y responsabilizarse del paciente; priorizar los requerimientos diagnósticos y terapéuticos de un paciente sobre cualquier otro interés; acompañar al enfermo terminal y brindar apoyo a la familia; ser crítico y reflexivo sobre sus acciones con los pacientes; y ser crítico y honesto con sus restricciones como internista para definir hasta dónde puede actuar y cuándo requiere de la acción de un internista subespecialista.

Administrativas y de gestión: optimizar los recursos del sistema de salud para visibilizar la práctica del internista como un especialista importante para el Sistema General de Seguridad Social en Salud (SGSSS); saber la normatividad que rige el SGSSS; y saber sobre la seguridad y la calidad de atención al paciente.

Pedagógicas como educador: ser educador del paciente y su familia y ser educador de otras generaciones de médicos y otros profesionales de la salud.

En investigación: realizar estudios epidemiológicos sobre enfermedades prevalentes en Colombia; conocer y practicar a diario la medicina basada en la evidencia; realizar una lectura crítica de la literatura científica y médica para categorizarla y aprovecharla; y generar preguntas de investigación a partir de los casos clínicos cotidianos.

De otra parte, según el análisis documental de dos programas de medicina interna, el perfil actual de formación de los residentes, futuros internistas del país, está previsto por objetivos y contenidos fragmentados; es decir, no están estructurados por CP. Sin embargo, se infiere que de forma empírica la formación se encuentra centrada en las $\mathrm{CP}$ específicas clásicas o clínicas para brindar la atención y el cuidado al paciente, con un modelo pedagógico tradicional y centrado en el "saber conocimientos médicos" y "el saber hacer"; no obstante, se han adelantado algunas actividades para desarrollar CP transversales en investigación y, además, se han organizado módulos teóricos para otras CP transversales emergentes, en uno de los dos programas curriculares.

Del análisis de las posibles relaciones entre el perfil de CP propuesto y el perfil de formación actual de esta especialidad, en el país, se concluye que:

1. Las CP específicas nucleares no están visibles y delimitadas en los programas curriculares.

2. Se da énfasis para que un residente desarrolle CP para la atención al paciente enfermo, por esto surgió la competencia profesional, enfatizar en la práctica de promoción de la salud y prevención de la enfermedad.

3. Algunas CP específicas clínicas no están explícitas, por ejemplo: saber hacer un examen físico completo y enfocado; construir un enfoque diagnóstico y terapéutico acorde a cada caso clínico; y tener juicio clínico.

4. En los documentos de los programas, tampoco están explícitas las CP para saber hacer diferentes procedimientos de diagnóstico y tratamiento.

5. De igual forma, no están explícitas las CP transversales emergentes con excepción de los avances en las competencias sobre investigación, pero con algunas limitaciones puesto que no es claro el tipo y el nivel de investigación que debe realizar un médico residente en una maestría de profundización, como lo es la especialidad de medicina interna (20); de todas formas se plantean algunos módulos teóricos sobre otras CP transversales en uno de los dos programas analizados.

6. Además, en los documentos está ausente el soporte pedagógico y epistemológico que aclare cómo se comprende el desarrollo del conocimiento médico y su aprendizaje; asimismo, falta hacer visibles los procesos que realiza un residente para aprender y actualizarse; de igual forma, no se presenta cómo un residente integra este conocimiento 
al desarrollo de capacidades para atender un paciente, es decir, a sus CP como futuro médico internista.

\section{Discusión}

Esta investigación se desarrolló para adelantar el camino de la construcción de perfiles profesionales en ciencias de la salud, fundamentados en un modelo pedagógico constructivista, en particular en las especialidades médicas y de éstas en la especialidad de medicina interna, para que los médicos internistas puedan responder a las exigencias actuales de esta profesión en Colombia y en el mundo como lo argumenta (Goñi, 2005) "resulta perentorio una visión mucho más integral y social de la educación universitaria [...] necesitamos personas activas y comprometidas en el medio social" (p. 100) (7); por esto ahora, el perfil de CP para un profesional de la medicina interna, debe estar enmarcado en el contexto científico, educativo, histórico, normativo, social y tecnológico.

En la investigación de Velásquez et al. (2008) de tipo descriptivo, mediante una encuesta virtual, en la que participaron 17 de los 21 coordinadores de los programas de medicina interna, que estaban funcionando para el año 2005 en el país, se indagó sobre el perfil de formación del residente de medicina interna en Colombia, encontraron que la formación del futuro especialista está centrada en la atención del paciente "intrahospitalario (69\%), mientras que sólo 31\% del tiempo se dedica a la atención de paciente ambulatorio" (p. 49); sumado a lo anterior, existen debilidades en lo relacionado con las actividades que desarrolla un residente en investigación, en las relacionadas con tratamiento paliativo, geriátrico y oncológico, a su vez, están ausentes las actividades para desarrollar competencias pedagógicas (capacidades como docente, en evaluación, en estrategias didácticas, entre otras). Además, tan sólo $12 \%$ de los coordinadores mencionaron que trabajan en temas del humanismo; tan sólo $18 \%$ mencionaron formación en "habilidades para administración de recursos, comportamiento ético, alta calidad científica, pensamiento y juicio crítico" (p. 50) (21).

En la anterior investigación los coordinadores respondieron que el tipo de internista que se pretende formar debe ser competente para actuar en los diferentes niveles de atención; además, se reveló que la formación del residente continúa siendo con un modelo pedagógico tradicional por contenidos y asignaturas fragmentadas con déficit de formación humanística y limitaciones en la evaluación para el aprendizaje. En este mismo sentido, el análisis documental, en la presente investigación, de dos programas de medicina interna en el año 2011, para revelar el perfil actual de formación de los futuros internistas, sigue siendo por objetivos y contenidos fragmentados como la situación encontrada en la investigación de Velásquez et al. (21). De esta forma, se corroboró que el panorama de los programas en esta especialidad, no ha cambiado sustancialmente; vale mencionar que para este proyecto el compromiso era hacer entrevista al menos a dos coordinadores y analizar dos programas. Sin embargo, se puede seguir explorando, para conocer si en otros programas han surtido cambios, lo importante es optimizar la formación de internistas o médicos de adultos.

Sin embargo, los relatos de los entrevistados, los aportes de los encuestados y la revisión de la literatura muestran que desde comienzos de la especialidad en medicina interna, en los años sesenta, los maestros apoyaban la formación del residente, de forma empírica, centrada en $\mathrm{CP}$ específicas clínicas para la atención al paciente, situación que perdura hasta el día de hoy. En contraste, los participantes aclararon que han surgido cambios trascendentales en la práctica profesional de la medicina interna, en Colombia dados por la tecnología aplicada al diagnóstico y al tratamiento, el auge de la investigación clínica, el desarrollo de la epidemiología clínica, la práctica clínica apoyada en la medicina basada en la evidencia (MBE), la revolución en las comunicaciones con la aparición de Internet y las bibliotecas virtuales que han inducido un cambio en las metodologías de enseñanzaaprendizaje; todos estos desarrollos inducen y aceleran el cambio en la profesión del médico internista y, por ende, en las CP que este especialista necesita desarrollar para tener un desempeño idóneo.

Al mismo tiempo la situación se ha hecho más compleja, en el contexto colombiano, porque se creó la Ley 100 en 1993 que llevó a repensar el quehacer de todos los profesionales de la salud, incluidos los médicos internistas, porque cambió el estilo de relación médico-paciente, o sea, se cambió el tipo de contrato social por el tiempo que tiene un internista disponible para cada paciente en especial en consulta externa, la dificultad de la continuidad del seguimiento de un paciente por la terminación de los contratos de servicio (22). Pero ante esta situación los participantes aclararon que lo importante son las CP de profesionalismo, de cualquier médico internista en todo nivel de atención y en cualquier escenario, puesto que un internista crea una relación con un paciente para elaborar soluciones a sus problemas; es decir, el profesionalismo lleva "implícito un compromiso de servicio al paciente y a la sociedad, no importa cuál sea la modalidad de aseguramiento o pago" (p. 86) (23). Sin embargo, toda la problemática que ha causado la Ley 100 de 1993, en el ejercicio de la medicina interna surgió de forma tangencial en esta investigación, puesto que se tenían otros objetivos, pero quedó claro que este sistema de "atención gerenciada de la salud" (24), en la que se perdió la visión de un paciente porque se cambió por la de "un cliente", atenta contra el ejercicio de la medicina interna al violentarse la autonomía del profesional, la continuidad de la relación médico-paciente y la toma de decisiones de diagnóstico y tratamiento (25).

Con relación a las CP específicas son precisas y clásicamente han sido explícitas, pero, al día de hoy, sólo algunas se están desarrollando en los programas de medicina interna, e incluso, algunas que son esenciales están invisibles, como: la capacidad de hacer una historia clínica integral, que trascienda hasta el aspecto humano, quizás como se enseñaba 
en los años sesenta cuando floreció esta especialidad en Colombia, con maestros internistas modelo.

En lo referente a las CP genéricas o transversales, que por definición son comunes a los profesionales de ciencia de la salud, están integradas por conocimientos, habilidades y actitudes como cualquier otra competencia profesional $(9$, 15, 26). Merece resaltarse la capacidad de comunicación de un médico y con su paciente como persona enferma, quien debe ser escuchado al elaborar la historia clínica a cabalidad; en esta circunstancia el individuo como paciente está reducido a una condición de dependencia, que será mayor según la severidad de su enfermedad $(25,27)$; porque cuando un enfermo ha perdido parte o casi toda su autonomía, requiere de un profesional competente que ante todo mantenga una excelente relación con él y su familia y sepa conjugar la diversidad de CP específicas, como las cuidado y atención al paciente, con las CP de comunicación (gestual, oral y escrita), no sólo con el enfermo y su familia sino con los demás profesionales y la sociedad.

En esta mirada holística sobre la formación del internista, también están otras $\mathrm{CP}$ transversales de profesionalismo que son un conjunto de principios éticos y deontológicos, valores o virtudes que definen la actuación, a su vez marcan la conciencia moral para que el especialista considere al enfermo como persona y no como un objeto por utilizar, como si fuese sólo un "libro de medicina interna". El profesionalismo, se entiende como un "conjunto de valores, principios y normas de actuación con las cuales se ha de ejercer una profesión" (p. 19) (28); en este caso, el internista es un profesional dispuesto a servir al ciudadano, en la posición de paciente, quien confía y "se pone en las manos de su médico"; por esto, la sociedad le da un estatus y un reconocimiento social, un internista no puede dejar interponer intereses propios o de otros, por encima de la empatía y el compromiso con el paciente. Entonces, la mejor opción de atención al paciente va desde la educación, la prevención, el diagnóstico y el tratamiento oportuno al elaborar soluciones específicas, particulares, pertinentes y oportunas $(3,27,29-31)$.

En este sentido, la relación médico-paciente que se desarrolla en la entrevista clínica es para que el internista construya la historia clínica del enfermo, de ahí que siempre los maestros "han insistido: no hay enfermedades sino enfermos, es decir, que las enfermedades tienen características particulares en cada individuo" (32). En cualquier entrevista hay un inicio, un desarrollo y un cierre; el primer paso es saludar al paciente y al familiar que está presente, para brindar un ambiente cálido y receptivo; al finalizar la sesión, el internista debe cerciorarse si el paciente y la familia comprendieron el diagnóstico, la indicación de los exámenes y el tratamiento. Por tanto, la actitud de escucha empática se expresa con lo verbal pero también con "la mirada y la gestualidad del profesional, que logra comprender las emociones del paciente" no sólo con interés científico, sino también con interés humano (p. 38) (32). En conclusión, como lo afirmó un participante, un internista requiere actuar con "empatía y capacidad de entender al otro, de ponerse en los zapatos del otro", por tanto, es prioritario que en los programas se dé un espacio real para que los residentes puedan desarrollar $\mathrm{CP}$ genéricas o transversales al aprender el conocimiento, las habilidades y las actitudes que las integran.

Esta propuesta de formación del residente, tiene como eje las CP nucleares que explican la razón de ser, es decir, la identidad deontológica de un internista, es allí dónde está la esencia del porqué el sistema de salud en Colombia, en crisis, requiere hoy en día, más que nunca, que las universidades sean propositivas con la construcción de currículos (con un modelo pedagógico y un plan de estudios) que favorezcan a los residentes para que desarrolle su identidad como internistas generales o médicos de adultos. Entonces, se comprende porque, es trascendental hacer pensar a las nuevas generaciones de residentes que sí es importante llegar a ser un internista, porque es socialmente digno, incluso, enriquecedor y satisfactorio para servir al paciente y la sociedad colombiana. De otra parte, es importante que los directivos y profesores formadores de internistas comprendan la esencia de la prevención primaria, tanto como la secundaria y la terciaria, para las enfermedades prevalentes en Colombia y el mundo (33). Queda así, una propuesta para fomentar la renovación de la formación de internistas, como fue concebido en los albores del siglo XX, en diversos países y continentes (15, 34-39).

Así, con la propuesta que se construyó en esta investigación se da respuesta a la especificidad del entorno colombiano para organizar la medicina interna como profesión y, por tanto, el perfil del egresado, la cual debe ser discutida y consensuada por las comunidades académicas y los actores de las universidades con su autonomía universitaria. Lo anterior implica una renovación del enfoque de los procesos de enseñanza, evaluación y aprendizaje de un residente, es decir, ahora se debe evaluar de acuerdo con lo que se busca que el estudiante apropie, en concordancia, no sólo se evalúan contenidos sino habilidades y actitudes que constituyen las CP de este especialista clínico (40-44). Además, con esta investigación se plantea una reflexión sobre la acción y en la acción, para la autorreflexión y la autorregulación, de las comunidades académicas y las universidades formadoras de internistas $(45,46)$.

Por lo anterior, quedan planteados algunos interrogantes sobre el marco legal de la acreditación de programas de pregrado y posgrado en medicina, en particular en la especialidad de medicina interna, así como la mirada crítica de los estándares de calidad expuestos en el Decreto 1665 de 2002, de los programas de Especializaciones Médicas y Quirúrgicas, en los que se trazó como meta el desarrollo de competencias para los médicos residentes (47).

Para finalizar, esta investigación da aportes al reconocer el papel de la pedagogía y la didáctica en los programas de posgrado de medicina; en particular, al visibilizar la complejidad de las $\mathrm{CP}$ que un residente requiere desarrollar para su desempeño idóneo como internista. La reflexión y la 
discusión de estos resultados servirán de apoyo a directivos y coordinadores de posgrado en especialidades médicas, para la toma de decisiones, en universidades privadas y públicas del país y Latinoamérica (48).

\section{Conclusiones}

Se reorganizó y enriqueció el perfil teórico de la literatura al construir un perfil de formación para internistas para Colombia, integrado por:

1. La primera familia emergente de $\mathrm{CP}$ específicas nucleares que dan la identidad deontológica a este médico especialista clínico, denominado médico internista, en otras palabras, éstas explican por qué este especialista es crucial, el director de orquesta, líder del equipo de atención al paciente adulto en cualquier sociedad y sistema de salud $(37,42,48)$.

2. Las CP específicas clásicas para brindar atención y cuidado al paciente son esenciales, pero surge el papel del internista en las actividades de prevención y promoción de la salud del adulto $(33,49,50)$.

3. La segunda familia emergente de $\mathrm{CP}$ transversales, que se denominan así, porque son comunes a los profesionales de ciencias de la salud: comunicativas; de profesionalismo y ética; administrativas y de gestión; pedagógicas como educador y en investigación $(48,51)$.

Es de aclarar que toda la literatura médica nacional e internacional propone las "competencias de conocimiento médico"; sin embargo, luego de la construcción conceptual de lo que es una competencia y una competencia profesional, en este proyecto de investigación se concluye que el conocimiento médico hace parte de todas las CP de un internista, las cuales evidencia como profesional al brindar atención y cuidado al paciente adulto.

El perfil de formación actual del residente continúa centrado en la atención al paciente "saber hacer" y en el aprendizaje de conocimiento médico sin el desarrollo formal de las CP específicas (clásicas y nucleares emergentes) y transversales; asimismo, la formación actual del residente continúa centrada en objetivos y contenidos que se revisan en las rotaciones o asignaturas de medicina interna y las subespecialidades (por ejemplo: consulta externa, urgencias, pisos, unidad de cuidado intensivo-UCI-, etc.). Sin embargo, se infiere un enfoque empírico de desarrollo de algunas CP específicas clásicas llamadas clínicas, para brindar atención y cuidado a un paciente, mediante los convenios docencia-servicio $(52,53)$. Los contenidos son esenciales en un plan de estudios; sin embargo, el modelo pedagógico vigente de esta especialidad médica es academicista porque fragmenta la realidad de la medicina interna en un sinnúmero de especialidades que incluso, algunas veces, se propone al residente opciones controversiales para crear las soluciones a los problemas de un paciente; es decir, no se le brinda un espacio, en lugar y tiempo, para integrar conocimiento, habilidades y actitudes, con el apoyo de equipos interdisciplinarios (25).
Además, del análisis documental de los programas actuales, se concluye:

1. Las CP específicas nucleares no están visibles.

2. Se da énfasis en la formación de los residentes centrada en la atención a pacientes, se puede inferir el desarrollo de CP clínicas.

3. Falta precisar algunas de estas CP específicas clínicas como saber hacer un examen físico completo y enfocado; construir un enfoque diagnóstico y terapéutico acorde a cada caso clínico que son fundamentales para desarrollar un juicio clínico.

4. Además, no están plasmadas en los documentos de los programas, las CP específicas para saber hacer diferentes procedimientos de diagnóstico y tratamiento.

5. De igual forma, no están expuestas las CP genéricas o transversales emergentes, con excepción de los avances en investigación con algunas dificultades, y algunos módulos teóricos sobre otras $\mathrm{CP}$ transversales, por ejemplo, en pedagogía y administración. Por tanto, es prioritario pensar sobre lo que es un currículo, acorde con un modelo pedagógico y un plan de estudios, en consecuencia, una postura epistemológica sobre lo que es ciencia, que va de la mano, con la razón de ser de la investigación médica en Colombia (25).

El perfil de $\mathrm{CP}$ propuesto para un médico internista en Colombia responde a las expectativas de un paciente, la normatividad vigente en la sociedad colombiana, el desarrollo de la medicina interna integral y las exigencias de la globalización. Además, se invita a la comunidad académica nacional formadora de médicos especialistas a pensar en la formación de los residentes para que lleguen a ser competentes frente a las necesidades de atención en salud; en particular, a la comunidad de medicina interna: directivos, profesores, médicos internistas y médicos residentes a reflexionar de forma crítica con el fin de optimizar su desempeño profesional y el de los futuros egresados; pero ante todo, insta a reconocer la identidad deontológica, la razón de ser de un internista respaldada por las CP nucleares, que explican el papel crucial de este especialista en el sistema de salud y la sociedad colombiana.

\section{Agradecimientos}

A la Universidad Nacional de Colombia. A los doctores Fabio Jurado Valencia, Ignacio Abdón Montenegro Aldana, Alberto Calderón García quienes fueron jurados. Al Dr. Tiburcio Moreno Olivos, de la Universidad Autónoma Metropolitana-Cuajimalpa (UAM-C) México D. F. A todos los participantes en este proyecto: directivos, médicos internistas, médicos internistas docentes y médicos residentes quienes en forma espontánea y oportuna decidieron dedicar su tiempo para aportar ideas, relatos y sugerencias valiosas.

\section{Referencias}

1. Palsson R, Kellett J, Lindgren S, Merino J, SempleC. \& Sereni, D. Interna medicine in Europe. Core competencies of the European internist: A discussion paper. Eur J Intern Med 2007; 18: 104-08.

2. Pinilla AE. Evaluación del aprendizaje en la educación superior. En: Pinilla AE, (Ed.). Reflexiones en Educación Universitaria. Primera edición. Bogotá: Unibiblos; 1999. p. 177 - 85.

3. Bogoya D. Del enfoque de contenidos al enfoque de competencias. En: Zerda A, Bejarano P y Orjuela F. (Eds.). La educación superior: tendencias, debates y 
retos para el siglo XXI. Sostenibilidad y financiación. Cátedra Manuel Ancizar. Bogotá: Editorial Universidad Nacional de Colombia; 2009. p. 391-406.

4. Cano H. La evaluación por competencias en la educación superior. Profesorado Revista de curriculum y formación del profesorado 2008; 12: 1-16.

5. Denyer M, Furnemont J, Poulain R, Vanloubbeeck G. Las competencias en la educación. Un balance. Primera edición. México. D.F.: Fondo de Cultura Económica; 2007.

6. Pinilla AE. Las competencias en la educación superior. En: Madiedo N, Pinilla AE, Sánchez J. (Eds.). Reflexiones en educación universitaria II: Evaluación. Segunda edición. Bogotá: Editorial Facultad de Medicina, Universidad Nacional de Colombia; 2008. p. 97-129.

7. Goñi JM. Las competencias. En: El espacio europeo de educación superior, un reto para la universidad. Competencias, tareas y evaluación, los ejes del currículum universitario. Barcelona: Octaedro; 2005. p. 85-120.

8. Jurado F. El enfoque sobre competencias: Una perspectiva crítica para la educación. Revista Complutense de Educación 2009; 20: 343-54.

9. Pinilla AE. Aproximación conceptual a las competencias profesionales en ciencias de la salud. Rev Salud Pública 2012; 14(5): 52-64.

10. Páramo P, Otálvaro G. Investigación alternativa: por una distinción entre posturas epistemológicas y no entre métodos. Cinta de Moebio. Revista de Epistemología de Ciencias Sociales 2006; 25: 1-8.

11. Bonilla-Castro E, Rodríguez P. El conocimiento científico de la realidad social. En: Más allá del dilema de los métodos. La investigación en ciencias sociales. Tercera Edición. Bogotá: Grupo Editorial Norma; 2000. p. 47-76

12. StraussA, Corbin J. Bases de la investigación cualitativa: técnicas y procedimientos para desarrollar la teoría fundamentada.Zimmerman E. (Trad.), Medellín: Editorial Universidad de Antioquia; 2002.

13. Páramo P y Gómez M. Confiabilidad y validez. En: Páramo P. (Comp.). La investigación en ciencias sociales. Técnicas de Recolección de información. Bogotá D. C.: Universidad Piloto de Colombia, Net Educativa; 2008. p. 47-52.

14. Álvarez-Gayau JJ. Cómo hacer investigación cualitativa. Fundamentos y metodología. México: Ediciones Paidós Ibérica. S.A.; 2010. p. 13-38.

15. Pinilla AE. Recuperando la identidad del médico internista. Al visualizar las CP. Acta Med Colomb 2010; 35: 143-53.

16. Muñoz-Justicia J, Sahagún-Padilla MÁ. Análisis cualitativo asistido por ordenador con Atlas.ti. En: Izquierdo C, Perinat A. (Eds.).Investigar en Psicología de la Educación. Nuevas Perspectivas Conceptuales y Metodológicas Barcelona: Amentia. p. 299-363. [Citado el 17 de julio de 2013] Disponible en: http://psicologiasocial.uab.es/juan/index.php/investigacion-mainmenu-42/publicacionesmainmenu-45

17. Coffey A, Atkinson P. Encontrar sentido a los datos cualitativos. Estrategias complementarias de investigación. Zimmerman, E. (Trad.), Medellín: Editorial Universidad de Antioquia; 2003.

18. Páramo P, Arango M. Cuestionarios. En: Páramo P (Comp.). La investigación en las ciencias sociales. Técnicas de recolección de información. Bogotá D.C.: Universidad Piloto de Colombia, Net Educativa. 2008. p. 55-72.

19. Resolución No 008430 de 1993 del 4 de octubre de 1993. Citado el 15 de julio de 2013. Disponible en: http://www.dib.unal.edu.co/promocion/etica_res_8430_1993.pdf

20. Decreto 1279 de junio 19 de 2002. Diario Oficial 44.840. Régimen salarial y prestacional para los docentes de las universidades estatales. [Citado el $17 \mathrm{de}$ julio de 2013]. Disponible en: http://viceacademica.univalle.edu.co/documentos/ normatividad/dec_1279.pdf

21. Velásquez JC, Pinilla AE, Pinzón A, Severiche D, Dennis R, Niño de Arboleda MN. Perfil de formación del posgrado de Medicina Interna en Colombia. Acta Med Colomb 2008; 33: 45-57.

22. Ley 100 de 1993. Por la cual se crea el sistema de seguridad social integral y se dictan otras disposiciones. [Citado el 20 de noviembre de 2011]. Disponible en: http://www.comisionseptimasenado.gov.co/salud/SALUD\%20EN\%20LEY\%20 $100 \% 20 \mathrm{DE} \% 201993$.pdf

23. Patiño JF. Humanismo, medicina y ciencia. En: Silva G (Ed.). Bogotá: Colección Obra Selecta. Editorial Universidad Nacional de Colombia; 2011.

24. Patiño JF. La desprofesionalización de la medicina en Colombia. Acta Med Colomb 2009; 34 (2): 271-77.

25. Pinilla AE. Modelos pedagógicos y formación de profesionales en el área de la salud. Acta Med Colomb 2011; 36: 204-18.

26. Baños JE, Pérez J. Cómo fomentar las competencias transversales en los estudios de Ciencias de la Salud: una propuesta de actividades. Educ Med 2005; 8: 216-25.

27. Vélez JR, Casasbuenas J. La historia clínica. Un ensayo sobre el acto sumo del arte e la medicina. Sus elementos prácticos y sus fundamentos filosóficos. Bogotá: Prisma Asociados; 2006.

28. Escudero JM, Vallejo M, Botías F. El asesoramiento en educación ¿Podrían ser las competencias profesorado una contribución positiva?. Revista de Curriculum y Formación de Profesorado 2008; 12: 1-23.

29. Torres H. ¡Humanicemos al internista!. El compromiso es de todos. Acta Med Colomb 2006; 31: 427-32.

30. Sarasti H. Qué es un internista. Acta Med Colomb 1980; 5: 415-22.

31. Rodríguez JJ. Definición de Profesión médica, Profesional médico/a y Profesionalismo médico. Educ Med 2010; 13: 63-6.

32. Vidal y Benito MC. La relación médico-paciente. Bases para una comunicación a medida. Buenos Aires: Lugar Editorial; 2010.

33. Nebot C, Rosales C, Borrell RM. Desarrollo de Competencias en atención primaria de salud. Rev Panam Salud Pública 2009; 26 (2): 176-83.

34. Duclos J. El internista: ¿una filosofía de vida?. Rev Méd Chile 2006; 134: 1219 1220.

35. Esguerra R. El médico para el nuevo milenio. Acta Med Colomb 1999; 24: 5-14.

36. Espinosa A. Medicina Interna ¿qué fuiste, qué eres, qué serás? Rev Cubana Med 1999; 38: 79-90.

37. Larson EB, Fihn SD, Kirk LM, Levinson W, Loge RV, Reynolds E, et al. The future of general internal medicine. Report and recommendations from the Society of General Internal Medicine (SGIM) Task Force on the Domain of General Internal Medicine. J Gen Intern Med 2004; 19: 69-77.

38. Sánchez CE. El internista del siglo XXI. Acta Med Colomb 1997; 22: 250-4.

39. Reyes H. ¿Qué es Medicina Interna?. Rev Méd Chile 2006; 134: 1338-44.

40. Beneitone P, Esquetini C, González J, Marty M, Siufi G, y Wagenaar R. Reflexiones y perspectivas de la Educación Superior en América Latina. Informe final. Bilbao, España: Universidad de Deusto y Universidad de Groningen 2007. Proyecto Alfa Tuning en América Latina 2004-2007. [Citado el 11 de abril de 2009] Disponible en:http://tuning.unideusto.org/tuningal/index.php?option=com docman\&Itemid=191\&task=view_category \&catid=22\&order=dmdate published\&ascdesc $=$ DESC

41. Epstein RM, Hundert EM. Defining and assessing professional competence. JAMA 2002; 287: 226-35.

42. LetelierLM, Valdivieso DA, Gazitúa R, EchávarriS, Armas, R. Definición actual de la medicina interna y el internista. Rev Méd Chile 2011; 139: 1081-88.

43. Federación Europea de Medicina Interna, el American College of Physicians, American Society of Internal Medicine (ACP-ASIM) y el American Board of Internal Medicine. Profesionalismo médico en el nuevo milenio: una declaración para el ejercicio de la medicina. (2010). Ann Intern Med 2002; 136: 243-6.

44. Pinilla AE. Evaluación de competencias profesionales en salud. Rev Fac Med 2013; 61: 47-64.

45. Perrenoud P. La construcción de una postura reflexiva a través de un procedimiento clínico. En: Desarrollar la práctica reflexiva en el oficio de enseñar. $2^{\mathrm{a}}$ reimpresión. Barcelona: Graó; 2010. p. 103-13.

46. Shön DA. La enseñanza del arte a través de la reflexión en la acción. En: La formación de profesionales reflexivos. Hacia un nuevo diseño de la enseñanza y el aprendizaje en las profesiones. Madrid: Paidós; 1987. p. 33-48.

47. Decreto 1665 de 2002. Estándares de calidad de los programas de Especializaciones Médicas y Quirúrgicas en Medicina. [Citado el 4 de abril de 2012] Disponible en: http://www.mineducacion.gov.co/1621/articles-86431_Archivo_pdf.pdf

48. Pinilla AE. Medicina Interna y Perfil del Internista. En: Archila PE, Senior JM (Eds.). Texto de Medicina Interna. Aprendizaje basado en problemas. Bogotá: Editorial Distribuna Editorial; p. 7-22.

49. Molina DI. Sistema General de Seguridad Social en Salud y ubicación labora de los médicos internistas en Colombia. En: Hernández C A, Ardila E y Niño de Arboleda MN. (Eds.). La medicina interna en Colombia. Bogotá: Asociación Colombiana de Medicina Interna; 2006. p. 46-79.

50. Ramírez ME, Castellanos-Suárez R, Badillo R, Naranjo FF, Insuasty JS, Gómez J, et al. El aprendizaje significativo de la medicina interna a través de la evaluación por competencias clínicas. Investigación-Acción. Salud UIS 2004; 36:111-24.

51. Schonhaut-Berman L, Millán-Klusse T, Hanne-Altermatt C. Competencias transversales en la formación de especialistas en pediatría, Universidad de Chile. Educ Méd 2009; 12: 33-41.

52. Ministerio de la Protección Social, Organización Panamericana de la Salud, Organización Mundial de la Salud. Consejo Nacional para el Desarrollo de los Recursos Humanos en salud. En: Modelo de evaluación de la relación docencia-servicio. Criterios básicos de calidad para centros de prácticas formativas. Bogotá; 2004. p. 7-12.

53. Decreto 2376 de julio 1 de 2010. Por medio del cual se regula la relación docenciaservicio para los programas de formación de talento humano del área de la salud. $M i$ nisterio de la Protección Social. [Citado el 16 de julio de 2013] Disponible en:http:// puj-portal.javeriana.edu.co/portal/page/portal/vice_academica/1documentos_vice/ Decreto\%20N\%FAmero\%202376\%201-jul-2010.pdf 